\title{
Clinically relevant CCR5 editing in primary CD4+ T cells with TALEN confers resistance to HIV-1 infection
}

\author{
Marianna Romito ${ }^{1}$, Alexandre Juillerat ${ }^{2}$, Yik Lim $\mathrm{Kok}^{3}$, Markus HIldenbeutel ${ }^{1}$, Manuel \\ Rhiel $^{1}$, Geoffroy Andrieux ${ }^{1}$, Johannes Geiger ${ }^{4}$, Carsten Rudolph ${ }^{4}$, Claudio Mussolino ${ }^{1}$, \\ Aymeric Duclert ${ }^{5}$, Karin Metzner ${ }^{3}$, Philippe Duchateau ${ }^{5}$, Toni Cathomen ${ }^{1}$, and Tatjana \\ Cornu $^{1}$ \\ ${ }^{1}$ University Medical Center Freiburg \\ ${ }^{2}$ Cellectis Inc. \\ ${ }^{3}$ University Hospital Zurich Division of Infectious Diseases and Hospital Epidemiology \\ ${ }^{4}$ ethris $\mathrm{GmbH}$ \\ ${ }^{5}$ Cellectis SA
}

May 19, 2020

\begin{abstract}
Today's available therapies to treat patients infected with human immunodeficiency virus (HIV) aim at preventing viral replication and transmission but fail to eliminate the virus. Although transplantation of an allogeneic CCR5 $\Delta 32$ homozygous stem cell grafts provided a cure for three patients, this approach is not considered a general therapeutic strategy because of potential severe side effects. Conversely, genome editing to disrupt the CCR5 locus that encodes the major HIV coreceptor was shown to confer resistance to R5-tropic HIV strains on the cellular level. Here, we present a clinically relevant and highly efficient approach to produce HIV-1 resistant CD4+ T cells. After transferring mRNA coding for CCR5-targeting TALEN into CD4+ $\mathrm{T}$ cells by electroporation, up to $89 \%$ of CCR 5 alleles were disrupted. Genotyping confirmed genetic stability of the edited cell product and off-target analyses established absence of relevant mutagenic events. When challenging these edited $\mathrm{T}$ cells with R5-tropic HIV, we observed protection in a dose-dependent manner. Functional assessments revealed no significant differences between edited and control CD4+ T cells in terms of proliferation capacity and their ability to secrete cytokines upon exogenous stimuli. Overall, we successfully engineered HIV-resistant CD4+ T cells under clinically relevant conditions, paving the way for clinical translation.
\end{abstract}

\section{Introduction}

As of today, there is no cure for infection with human immunodeficiency virus type 1 (HIV-1). The available antiretroviral therapies (ART) target different steps in the HIV replication cycle. Although ART manages to reduce HIV burden below detection limit as long as the medication is taken, it does not eradicate the virus. Inhibition of HIV entry has been considered a promising strategy that was explored in numerous ways. In particular, targeting CCR5, the co-receptor of prevalent HIV-1 strains, has been pursued using CCR5 inhibitors or blocking antibodies [1]. But again, even when combined with other ARTs, they do not eradicate HIV.

On the other hand, the knowledge that a naturally occurring 32 nucleotide deletion ([?]32) mutation in $C C R 5$ that abrogates CCR5 protein expression leads to resistance to R5-tropic HIV-1 strains [2] [3], has spurred some encouraging approaches [4]. People with a homozygous $\Delta 32$ mutation are clinically unremarkable and conduct a normal life [5]. The $\Delta 32$ allele can only be found in the Caucasian population and follows a specific distribution pattern in Europe. It was proposed that the higher allelic frequency of this mutation in 
Northern Europe of about $10 \%$ was due to smallpox infections in the Middle Ages: individuals lacking CCR5 had a higher chance of survival, leading to a strong selection for the $\Delta 32$ mutation in a few decades [6]. In 2007, an HIV-positive patient, who later was referred to as the Berlin Patient, was transplanted with an allogeneic stem cell graft of an HLA-matched donor homozygous for the CCR $\Delta 32$ mutation after diagnosis of acute myeloid leukemia (AML). After stopping ART, the patient was closely monitored for virus titers. As of today, i.e. 13 years later, no viral RNA or proviral DNA has been detected, so that he was declared the first patient to be cured from HIV [7-9]. Following the principle of the Berlin patient, recently two more patients, known as the London [10,11] and the Düsseldorf [12] patients, might be the next patients to be declared as cured. These observations made it clear that it is possible to transfer HIV resistance from a donor to a patient. However, due to the potential severe side effects that allogenic transplantations encompass and the difficulty to find matching homozygous $\Delta 32$ donors, this strategy is not considered a universal approach to treat patients affected by HIV.

Alternatively, CCR5 gene editing in autologous cells may represent a potent approach to provide patients with cells that are resistant to HIV [13]. To this end, genome editing with programmable nucleases, in particular zinc-finger nucleases (ZFN) and CRISPR-Cas nucleases, have been used in several preclinical studies to disrupt the CCR5 gene in T cells (reviewed in $[14,15]$ ). Moreover, in a clinical trial that enrolled 12 patients, a single dose of $C C R 5$-edited autologous CD4+ T cells were administered. Although the effect lasted only transiently, the blood level of HIV provirus decreased in most patients and the infusion of CCR5 -modified autologous CD4+ T cells proved to be safe [16].

In our study we sought to engineer HIV-1 resistant CD4+ $\mathrm{T}$ cells in a manner compatible with good manufacturing practice (GMP). We aimed at disrupting the CCR5 locus in those clinically relevant cell type by transferring mRNA that code for a highly effective transcription activation-like effector nuclease (TALEN) using a GMP compliant electroporation device. We have previously shown that TALENs can be employed to disrupt $C C R 5$ in human cell lines with high specificity $[17,18]$. Here, we show that improvement of the TALEN platform in combination with novel RNA transfer technologies [19] enabled us to knockout CCR5 in CD4+ T cells with high activity and high specificity. The manufactured T cells were HIV resistant, genetically stable, and maintained their proliferation capacity as well as their ability to respond to exogenous stimuli.

\section{Materials and methods}

CD4+ T cells cultivation and editing: CD4+ T cells and PBMCs were isolated from leukocyte reduction system (LRS) chambers obtained from the Blood Donation Center at the Medical Center - University of Freiburg after informed consent. CD4+ T cells were isolated using CD4 MicroBeads (Miltenyi, Germany), cultivated in X-Vivo15 (Lonza, Switzerland) supplemented with $20 \mathrm{U} / \mathrm{ml} \mathrm{IL-2} \mathrm{(Miltenyi,} \mathrm{Germany),} \mathrm{and}$ activated with the T Cell Activation/Expansion Kit (Miltenyi, Germany). After 3 days, beads were removed

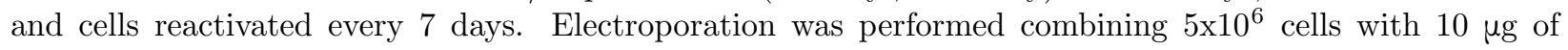
each TALEN mRNA or $5 \mu \mathrm{g}$ of chemically modified GFP mRNA [20]. Cells were electroporated in $100 \mu \mathrm{l}$ electroporation buffer using P3 Primary Cell 4D-Nucleofector kit (Lonza, Switzerland) and program EO-115, or in 200ul of BTXpress Cytoporation medium T (BTX, Harvard Bioscience USA) (1 pulse 1200 V, $0.1 \mathrm{~ms}$, $0.2 \mathrm{~ms}$ interval, 1 pulse $1200 \mathrm{~V}, 0.1 \mathrm{~ms}, 100 \mathrm{~ms}$ interval and 4 pulses $130 \mathrm{~V}, 0.2 \mathrm{~ms}, 2 \mathrm{~ms}$ intervals). After harvesting, genomic DNA was extracted with DNeasy Blood \& Tissue Kits (Qiagen, Netherlands). NGS or T7E1 assay was performed and analyzed as previously described [21].

mRNA production : For in vitro RNA transcription, $10 \mu \mathrm{g}$ of TALEN encoding plasmid DNA containing a T7 promoter and a poly(A) were linearized with HindIII-HF (NEB, Germany). The templates were purified using the QIAquick@ Gel Extraction Kit (Qiagen, Netherlands) and RNA transcribed with mMESSAGE mMACHINER T7 Ultra kit (Thermo Fisher Scientific, USA). The RNA pellets were resuspended in buffer EB (Qiagen, Netherlands) and stored at $-80^{\circ} \mathrm{C}$.

Lentiviral transduction : For production [22], HEK293T cells (ATCC) were grown in DMEM GlutaMAX I (Gibco, Life Technologies, USA) supplemented with 10\% FCS (GE Healthcare, UK), 10.000 U/ml penicillin 
(GE Healthcare, UK), $10 \mathrm{mg} / \mathrm{ml}$ streptomycin (GE Healthcare, UK) and $100 \mathrm{mM}$ sodium pyruvate (PAA Laboratories, Austria). $15 \times 10^{6}$ cells were seeded per $15 \mathrm{~cm}$ plate $24 \mathrm{~h}$ before transfection. Prior to transfection medium was supplemented with chloroquine 1:1000 (Sigma-Aldrich, Germany) and polyethyleneimine (PEI) transfection performed with $15 \mu \mathrm{g}$ pMDLg/pRRE (Addgene), $3 \mu \mathrm{g}$ pRSV-Rev (Addgene), $15 \mu \mathrm{g}$ of the lentivirus encoding plasmid, and $5.1 \mu \mathrm{g}$ of the envelope encoding plasmid (Bal_gp120, kind gift of Dr. Boris Fehse, or pMD2.G, Addgene). After $12 \mathrm{~h}$, transfection mix was exchanged with medium containing $10 \mathrm{mM}$ sodium butyrate (Sigma-Aldrich, Germany). Supernatants were harvested $32 \mathrm{~h}$ and $56 \mathrm{~h}$ post-transfection, centrifuged at $20000 \mathrm{xg}$ at $4^{\circ} \mathrm{C}$ for $2 \mathrm{~h}$ on a $20 \%$ sucrose cushion using a swingout rotor. Pellets were resuspended in $50 \mu \mathrm{l}$ of PBS and stored at $-80^{\circ} \mathrm{C}$. Lentiviral EGFP vectors were titrated on PM1 cells (NIH AIDS Reagent Program, Division of AIDS, NIAID, NIH [23]) that were cultivated in RPMI 1640 medium containing L-Glutamine (Gibco Life Technologies, USA), 10\% FCS (GE Healthcare, UK), 10.000 U/ml penicillin (GE Healthcare, UK) and $10 \mathrm{mg} / \mathrm{ml}$ streptomycin (GE Healthcare). 5x10 ${ }^{4}$ PM1 cells in medium supplemented with $4 \mu \mathrm{g} / \mathrm{ml}$ polybrene were transduced with serial dilutions of the vectors by spinoculation for $1 \mathrm{~h}$ at $200 \mathrm{xg}$ at $32^{\circ} \mathrm{C}$. For transduction, $5 \times 10^{4} \mathrm{CD} 4+\mathrm{T}$ cells were cultured in X-Vivo15 medium (Lonza, Switzerland) containing $4 \mu \mathrm{g} / \mathrm{ml}$ polybrene with 6 transducing units/cell by spinoculation for $1 \mathrm{~h}$ at $200 \mathrm{xg}$ at $32^{\circ} \mathrm{C}$.

Flow cytometry : GFP expression after lentiviral transduction and/or cell viability (7AAD, AppliChem, Germany) were assessed using BD Accuri C6 Flow Cytometer (BD Biosciences, USA). CCR5 expression was detected by labelling $1 \times 10^{5}$ cells in $50 \mu$ of PBS with $2 \mu$ of APC-labelled mouse anti-human CD195 antibody (BD Biosciences, USA) for $20 \mathrm{~min}$ at room temperature. Cells were analyzed on BD FACS Canto-II (BD Biosciences, USA).

HIV-1 infection: Full-length HIV-1 provirus encoding plasmids were obtained from NIH AIDS Research and Reference Reagent Program, Division of AIDS, NIAID, NIH [24, 25]. Virus stocks HIV-1 JR-FL (CCR5tropic) and $\mathrm{HIV}-1_{\mathrm{NL} 4-3}$ (CXCR4-tropic) were generated and titrated as previously described [26]. For infection cells were activated as described above, and $2 \times 10^{5} \mathrm{CD} 4+\mathrm{T}$ cells were infected with either HIV-1 JR-FL or $\mathrm{HIV}-1_{\mathrm{NL} 4-3}$ at MOIs of 0.01 and 0.001 . At indicated time points $50 \mu \mathrm{l}$ of cell culture supernatant were harvested and used to determine the amount of p24 by ELISA [27].

Cytokine release : $200 \mu \mathrm{l}$ of supernatants were harvested from $1 \times 10^{6}$ cells per sample at indicated timepoints. Cytokine concentrations were determined using Cytometric Bead Array plus beads and standards for each analyte: IFN- $\gamma$, IL-2 or TNF- $\alpha$ (BD Biosciences, USA). Cells were stimulated with CD3/CD28/CD2 beads (Miltenyi Biotec, Germany), $1 \mu \mathrm{g} / \mathrm{ml}$ ionomycin (Merck Millipore, Germany) or $10 \mathrm{ng} / \mathrm{ml}$ PMA (Sigma-Aldrich, Germany.

Off-target analyses : Potential off-target sites were predicted with PROGNOS web tool (http://bao.rice.edu/cgi-bin/prognos/prognos.cgi) using the TALEN v2.0 algorithm [28]. Five mismatches per half sites were allowed. Loci were PCR amplified using the primers listed in Table S1. Libraries were prepared using the NEBNext Ultra II DNA Library Prep Kit (NEB, Germany) and quantified using ddPCR Library Quantification Kit for Illumina TruSeq (Biorad, Germany). Samples were sequenced on an Illumina MiSeq platform using MiSeq Reagent Kit v2, 500-cycles (Illumina, USA), and data analyzed with Crispresso2 to establish significance and indel types [29] . $p$ values were adjusted with the Benjamini \& Hochberg [30] method. For oligonucleotide capture assay (OCA), human PBMCs (ALLCELLS, USA) were plated at a density of $1 \times 10^{6}$ cells $/ \mathrm{ml}$ in X-vivo-15 media (Lonza, Switzerland) supplemented with $5 \%$ human AB serum (Gemini, USA) and $20 \mathrm{ng} / \mathrm{ml}$ of IL-2 (Miltenyi, Germany). The next day, PBMCs were activated using human T activator CD3/CD28 (Thermo Fisher Scientific, USA). Four days later, 5x10 ${ }^{6} \mathrm{~T}$ cells in cytoporation medium $\mathrm{T}$ were electroporated with $20 \mu \mathrm{g}$ of TALEN mRNA (10 $\mu \mathrm{g}$ each of left and right subunit) and $10 \mu \mathrm{l}$ of pre-annealed oligodeoxynucleotides (dsODN, $100 \mu \mathrm{M}$ ) using an AgilePulse MAX system (Harvard Apparatus, USA) and a $0.4 \mathrm{~cm}$ cuvette. Genomic DNA was extracted 3 days later, randomly sheared to 300 bp fragments by sonication (Covaris LE220-plus), fragments end-repaired/A-tailed (NEBNext@ Ultra End Repair/dA-Tailing Module, USA), and next-generation sequencing Y-adapters (TruSeq Annealed Adapter) were added. Two rounds of anchored PCR using dsODN-specific and adapter-specific primers were per- 
formed. Adapter-specific (P5_1) and dsODN-specific primers were used in the first PCR. Adapter-specific (P5_2) primers, dsODN-specific primers $\mathrm{P} 7$ and primers adding the barcode and P7 sequence to the ends of the PCR product were used in the second PCR. PCR products were pooled and sequenced using Illumina NextSeq $(2 \mathrm{x} 150 \mathrm{bp})$. The resulting sequences reads were mapped to the human genome to identify potential off-target sites.

Statistical analyses : A time series analysis [31] was performed to compare the samples in both the HIV-1 challenge and growth curves experiments. Briefly, two cubic regression models were fitted to each growth curve. The full-model captures specific time course for each separate treatment whereas the reduced-model specifies a single set of parameters for two tested treatments. Finally, the goodness of the fit between the full and the reduced models was tested by Anova where $P$-value indicates the probability that the two models are the same. $P$-values are indicated with * [?]0,05; ** [?]0,001; ***[?]0,0001. For all other analyses, the Student's T-test was applied.

\section{Results}

\section{CCR5 knockout is genetically stable in $\mathrm{CD} 4+\mathrm{T}$ cells}

The target locus in exon 3 of the CCR 5 gene was chosen to be in the region coding for the $\mathrm{N}$-terminus of the CCR5 protein (Fig. 1A). The rationale was two-fold: (1) out-of-frame insertion/deletion (indel) mutations will cause frameshift mutations leading to early termination of translation, and (2) it encodes a conserved region of CCR5 that is bound by HIV-1 gp120. Even in-frame indels will remove critical residues necessary for HIV-1 to bind to CCR5 [32, 33]. We first established the optimal conditions to achieve high CCR5 disruption in CD4+ T cells from different donors with TALEN encoding mRNA via electroporation. Cells were cultivated for several days and subjected to T7E1 assay at days 7 and 21 of culture to monitor gene disruption frequencies. A transient cold shock increased allelic CCR5 disruption frequency from $50 \%$ to $85 \%$ (Fig. 1B). The indel frequencies ranged between 50-90\% at day 7 of culture and stabilized around $85 \%$ after 21 days, demonstrating stable editing in long term cultures. We also evaluated the effect of the gene disruption on the surface expression of CCR5. CCR5 expression varies between cell subsets and donors [34] as well as during activation, therefore staining was performed at the same culture stage. The percentage of CCR5 positive cells was significantly decreased $(p=0.0009)$ after editing with TALEN while no significant decrease in CCR5 expression was detected in control samples treated with only the left TALEN arm (Fig. 1C). Reduction of CCR5 protein expression remained significant $(p=0.0314)$ and stable for 21 days, which paired well with the observations made on the genomic level (Fig. 1B),

In parallel we edited cells from a $\Delta 32$ heterozygous donor. Allelic gene disruption rates reached $69 \%$ at day 7 and $86 \%$ at day 21 (Fig. 1B), so well within the ranges of the homozygous CCR5 wt donors. When performing a phenotypic analysis, a drop of $50 \%$ in CCR5 expression was also observed for this donor (Fig. $1 \mathrm{C}$, red dots), in line with the results seen with the homozygous donors.

In order to better understand the type of indels generated by the nucleases, we performed next generation sequencing (NGS) analysis on the edited CCR5 target locus in 3 different donors, one of which being $\Delta 32$ heterozygous, and analyzed the indels at days 7 and 21, respectively (Fig. 1D-E). The indel patterns and frequencies remained comparable between samples and over time. There was no significant enrichment or depletion of a specific indel type detected by a paired $t$-test during the culture period. This indicates that the clonal distribution of the edited cells between the two time points remained stable. In all 3 donors, the most frequent indel was a 19-nt deletion, followed by a 9-nt deletion, that combined represented about $20 \%$ of the total indel mutations. Of note, the four most frequent deletions $-19,-9,-13$, and -26 are likely due to microhomologies, as detected by the Microhomology-Predictor tool [35].

\section{CCR 5 edited CD4+ $\mathbf{T}$ cells are resistant to $\mathrm{HIV}-1$ infection}

As a proof of concept, we challenged the edited cells at an early and late culture time point with GFP expressing lentiviral vectors pseudotyped with either the gp160 glycoprotein of the HIV-1 R5-tropic strain BaL or VSV-G as control. The CCR 5 edited CD4+ T cells showed approximately $50 \%$ reduced susceptibility 
to transduction with the HIV-gp160 pseudotyped vector when compared to control cells (Fig. 2A). When challenging $\mathrm{T}$ cells from two $\Delta 32$ heterozygous donors, a decreased susceptibility - similar to cells from the other donors - was observed. As a CD4/CCR5-independent transduction control, we transduced the cells in parallel with VSV-G pseudotyped lentivectors. Under these conditions we did not see any alteration in transduction efficiency.

Next, we tested resistance of $C C R 5$ edited CD4+ T cells to replication-competent HIV-1 by infecting them

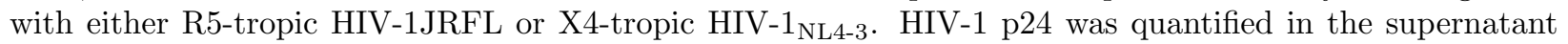
of infected cells as a measure of viral replication (Fig. S1A-B). In samples infected with HIV-1JRFL (high or low MOI), a significant protective effect $\left(p=8.8 \times 10^{-4}\right.$ and $\left.p=5.7 \times 10^{-5}\right)$ was observed when compared to infection of unedited cells. As expected, infections with X4-tropic HIV-1 $1_{\mathrm{NL} 4-3}$ did not reveal significant differences (Fig S1B). Furthermore, to investigate the impact of the editing rate on the viral replication, we spiked edited CD4+ T cells with unedited cells at ratios of 1:1 (50\% edited cells) and 1:3 (25\% edited cells). Low MOI infection with HIV-1JRFL, as determined by p24 in the supernatant, was significantly $(p$ $\left.=2.72 \times 10^{-14}\right)$ reduced in non-diluted edited cells $(100 \%)$ when compared to unedited samples (Fig. 2B). The protective effect was also observed in both the $50 \%$ and $25 \% \operatorname{mix}\left(p=8.38 \times 10^{-11}, p=1.13 \times 10^{-4}\right)$, suggesting that a cell population with $20 \%$ of edited $C C R 5$ alleles was able to slow down virus replication. A significant protective effect $\left(p=3.06 \times 10^{-3}\right)$ was also observed when edited cells $(100 \%)$ with infected with a high MOI (Fig. 2C), albeit to a lower degree as compared to the low MOI infection. After dilution (50\% mix), the protective effect was lost when cells were infected at high MOI $(p=0.98)$. In the control settings with HIV-1 $1_{\text {NL4-3 }}$ infections, we did not see any impairment of virus replication in the edited cells (Fig. 2D), as expected. We conclude that editing of CD4+ T cells with CCR5 targeting TALEN leads to protection against R5-tropic HIV-1 in an editing dose-dependent manner.

\section{CCR5 edited CD4+ $\mathbf{T}$ cells maintain potency}

Transfer of mRNA to $\mathrm{T}$ cells via electroporation can potentially affect cell viability and/or functionality. We addressed this point by evaluating viabilities $24 \mathrm{~h}$ post-treatment. CD4+ T cells electroporated with TALEN mRNA (both arms, or left arm only), GFP mRNA, or mock pulsed, showed a decrease in viabilities of $\sim 30 \%$ when compared to untreated samples (Fig. 3A), implying that the electric pulse but not the RNA transfer had an impact on $\mathrm{T}$ cell viabilities. On the other hand, the temperature shift did not affect viability.

We next wanted to investigate if CCR5 edited cells retain their proliferative potential. To this end, the edited CD4+ T cells and control samples were cultivated under proliferation activating conditions. The total number of cells at days 7, 14 and 21 was comparable for all tested conditions (Fig. 3B-C). A timeseries analysis [31] confirmed that the samples, independent of cultivation temperature or treatment, did not show significant differences in proliferation. Hence, we conclude that editing CCR 5 did not affected the expansion capabilities of CD4+ T cells.

To explore if editing $C C R 5$ has an impact on $\mathrm{CD} 4+\mathrm{T}$ cell function in terms of cytokine release upon stimulation, we stimulated the cells with either phorbol 12-myristate 13-acetate (PMA), ionomycin or CD3/CD28/CD2 to trigger the release of TNF- $\alpha$, IFN- $\gamma$ or IL2, respectively. Supernatants of electroporated $\mathrm{T}$ cells were harvested $8-48 \mathrm{~h}$ after stimulation and analyzed by cytometric bead array. While we observed variations in the amounts of secreted cytokines among the four donors, the values did not significantly (two-sided $t$-test) differ when comparing the various treatment regimens (Fig. 3D-F). Unstimulated cells did not release measurable amounts of cytokines. In sum, these results demonstrate that editing CCR5 did not impair the ability of the engineered CD4+ T cells to respond to the different stimuli.

\section{Editing CCR5 in CD4+ T cells with high specificity}

In order to assess the genotoxic potential of TALEN mediated editing of CCR 5 , we investigated the occurrence of off-target (OT) activity by two different means: targeted amplicon sequencing of in silicopredicted OTs or an unbiased in cellula assay. The top 20 potential OTs were predicted using the PROGNOS tool [28]. Amplicons from edited samples were compared to amplicons of untreated samples (Fig. 4A). Two of the top 20 OTs revealed some low but significant OT activity over background (Table S2). However, both 
OT01 ( $0.12 \%$ vs. $0.10 \%$ background, $p=0.000211)$ and OT10 (0.08\% vs. $0.07 \%$ background, $p=0.004422)$ are situated in intronic regions. These data were complemented with an unbiased OT detection approach termed oligonucleotide capturing assay (OCA) which is based on GUIDE-seq [36]. The highest score (OCA1) was obtained for the CCR5target site (Fig. 4B). While OT01 was also found by OCA (OCA03), OT10 did not match with any of top 24 OCA hits (Table S3). While CCR2did not come up as a potential OT in PROGNOS, OCA picked up a weak activity at CCR2 (OCA4). Importantly however, all identified OCA sites had a considerably lower score than the CCR5 on target site (OCA1). Together, these OT analyses demonstrate that the employed TALENs are highly specific designer nucleases with minimal OT activity.

\section{Discussion}

Approaches to disrupt CCR5 in CD4+ T cells have been previously described. These reports demonstrated the feasibility of abrogating HIV entry, albeit with some drawbacks including low gene disruption efficiencies, high off-target effects or absence of specificity analyses, missing potency analyses, and/or lack of GMP compatibility [37-40].

In this study, we present efficient editing of the CCR5 locus in primary CD4+ T cells in a GMP-compliant manner. Under these conditions, we disrupted up to $90 \%$ of $C C R 5$ alleles with no notable off-target activity, suggesting that the employed TALEN are highly specific. Disrupting the region that encodes the N-terminus of the CCR5 protein in CD4+ T cells gave rise to cells resistant to infection with R5-tropic HIV-1 but, as expected, not X4-tropic virus. Importantly, CCR5 edited CD4+ T cells did not show any differences to nonedited control cells in the applied proliferation and potency assays. This indicates that TALEN-mediated editing of CCR5 is efficacious and safe, opening a window for therapeutic applications.

Our data demonstrate that low CCR 5 editing frequency is not sufficient to abrogate HIV infection, putting forward that efficient disruption that mediates biallelic gene knockouts are paramount to see clinical effects [16]. This is in line with a recently published case report confirming that a CCR5 knockout frequency of $5 \%$ in the $\mathrm{T}$ cell compartment is not sufficient to achieve clinical benefit [41].

A crucial point for a clinical trial is to recruit patients exclusively positive for R5-tropic HIV variants, since the absence of CCR5 would favor the propagation of X4-tropic viruses if present in the patient. The importance of proper examination was seen in the 'Essen patient' [42] who suffered from an X4 rebound after allogenic transplantation of a $\Delta 32$ homozygous graft. In a CD4 $+\mathrm{T}$ transplantation scenario, performing a knockout of both coreceptor encoding loci $C C R 5$ and $C X C R 4$ can be considered. Strategies employing ZFN or CRISPR-Cas9 in CD4+ T cells conferred in fact resistance to R5- and X4-tropic viruses [43-45]. This would prevent a rebound of X4-tropic viruses, but concomitant generation of two DNA double strand breaks drastically increase the probability for translocations and thus the risks of malignancies.

It was recently discovered, that a lack of CCR5 is associated with worse clinical outcomes in West Nile Virus infections, although CCR5 is not playing a direct role in the infection cycle [46]. This implies that one should carefully assess the risks and benefits of applying CCR 5 edited cells in regions where West Nile Virus infections are prevalent.

A side effect of gene editing approaches is genotoxicity as a consequence of OT effects. OT activity can induce mutagenesis but also provoke chromosomal rearrangements, both potentially inducing malignancies. Employing highly specific designer nucleases is therefore paramount to mitigate the risk of such effects. Several tools are available to identify OT events, one of them being in silicopredictions. The reported studies $[37,39]$ analyzed off-target events using this tool with the drawback of having some biases, which leads to overseeing some important off-targets. Another study [38] performed off-target analysis using whole genome sequencing. This method does not reach a sufficient depth to detect rare events. In our study, we carefully evaluated OT using both in silico prediction as well as by employing an unbiased in vivo oligonucleotide capturing assay. We detected two rare OT events occurring in introns of genes, thus minimizing the risks of adverse effects. For instance, the OT site in CNOT10 (OT1), which was also detected by the OCA (OCA3), was cleaved in about $0.12 \%$ of cells. OCA identified three additional OT sites, one of them in the CCR2 gene. But again, the OCA scores of these OTs was similarly low as OCA3, suggesting OT activities in the 
range of $0.1 \%$ Moreover, targeting terminally differentiated cells, like CD4+ T cells, furthermore mitigates the risk of developing malignancies. Besides, all functional assays we performed in this study demonstrated that the CCR 5 edited cells behaved as unedited cells, suggesting that TALEN expression did not have any impact on these cells.

All in all, we show that we can manufacture high functioning edited CD4+ T cells with low genotoxicity that can be scaled up to clinical relevant numbers and be applied to patients.

\section{Acknowledgement}

We thank Boris Fehse and the NIH AIDS Research and Reference Reagent Program for providing plasmids, Kay Ole Chmielewski for help with OT analysis, Ilona Skatulla and Petra Scheliga-Marschner for technical support, the Lighthouse Core Facility (Medical Center - University of Freiburg) for flow cytometry support, and the Blood Donation Center (Medical Center - University of Freiburg) for providing PBMCs.

This work was supported by a grant of the German Federal Ministry of Education and Research (BMBF01EO0803 to T.I.C. and T.Ca.) and a funded research cooperation with Cellectis (ZVS20170614 to T.I.C. and T.Ca.).

\section{Conflict of interest}

A.J., A.D. and P.D. are employees of Cellectis. J.G. and C.R. are employees of Ethris. T.I.C. and T.Ca. have sponsored research collaborations with Cellectis and Miltenyi Biotec. K.J.M. received travel grants and honoraria from Gilead Sciences, Roche Diagnostics, GlaxoSmithKline, Merck Sharp \& Dohme, Bristol-Myers Squibb, ViiV and Abbott. The University of Zurich received advisory board honoraria from Gilead Sciences and research grants from Gilead Science, Roche, and Merck Sharp \& Dohme for studies in which K.J.M. serves as principal investigator.

\section{References}

1. Gilliam, B.L., D.J. Riedel, and R.R. Redfield, Clinical use of CCR5 inhibitors in HIV and beyond. J Transl Med, 2011. 9 Suppl 1 : p. S9.

2. Deng, H., et al., Identification of a major co-receptor for primary isolates of HIV-1. Nature, 1996. 381 (6584): p. 661-6.

3. Dean, M., et al., Genetic restriction of HIV-1 infection and progression to AIDS by a deletion allele of the CKR5 structural gene. Hemophilia Growth and Development Study, Multicenter AIDS Cohort Study, Multicenter Hemophilia Cohort Study, San Francisco City Cohort, ALIVE Study. Science, 1996. 273 (5283): p. $1856-62$.

4. Kalidasan, V. and K. Theva Das, Lessons Learned From Failures and Success Stories of HIV Breakthroughs: Are We Getting Closer to an HIV Cure? Front Microbiol, 2020. 11 : p. 46.

5. Huang, Y., et al., The role of a mutant CCR5 allele in HIV-1 transmission and disease progression. Nat Med, 1996. 2 (11): p. 1240-3.

6. Galvani, A.P. and M. Slatkin, Evaluating plague and smallpox as historical selective pressures for the CCR5-Delta 32 HIV-resistance allele. Proc Natl Acad Sci U S A, 2003. 100 (25): p. 15276-9.

7. Hutter, G., et al., Long-term control of HIV by CCR5 Delta32/Delta32 stem-cell transplantation. N Engl J Med, 2009.360 (7): p. 692-8.

8. Hutter, G. and E. Thiel, Allogeneic transplantation of CCR5-deficient progenitor cells in a patient with HIV infection: an update after 3 years and the search for patient no. 2. AIDS, 2011.25 (2): p. 273-4.

9. Allers, K., et al., Evidence for the cure of HIV infection by CCR5Delta32/Delta32 stem cell transplantation. Blood, 2011.117 (10): p. 2791-9. 
10. Gupta, R.K., et al., HIV-1 remission following CCR5Delta32/Delta32 haematopoietic stem-cell transplantation. Nature, 2019. 568 (7751): p. 244-248.

11. Gupta, R.K., et al., Evidence for HIV-1 cure after CCR5Delta32/Delta32 allogeneic haemopoietic stemcell transplantation 30 months post analytical treatment interruption: a case report. Lancet HIV, 2020.

12. B.-E., J., et al., Analytic treatment interruption (ATI) after allogeneic CCR5-D32 HSCT for AML in 2013. Proceedings of the Conference on Retroviruses and Opportunistic Infections (CROI), Seattle, WA, 2019.

13. Cornu, T.I., et al., Editing CCR5: a novel approach to HIV gene therapy. Adv Exp Med Biol, 2015. 848 : p. $117-30$.

14. Cornu, T.I., C. Mussolino, and T. Cathomen, Refining strategies to translate genome editing to the clinic. Nat Med, 2017.23 (4): p. 415-423.

15. June, C.H., Emerging Use of CRISPR Technology - Chasing the Elusive HIV Cure. N Engl J Med, 2019. 381 (13): p. 1281-1283.

16. Tebas, P., et al., Gene editing of CCR5 in autologous CD4 T cells of persons infected with HIV. N Engl J Med, 2014.370 (10): p. 901-10.

17. Mussolino, C., et al., TALENs facilitate targeted genome editing in human cells with high specificity and low cytotoxicity.Nucleic Acids Res, 2014.

18. Mussolino, C., et al., A novel TALE nuclease scaffold enables high genome editing activity in combination with low toxicity. Nucleic Acids Res, 2011. 39 (21): p. 9283-93.

19. Gautron, A.S., et al., Fine and Predictable Tuning of TALEN Gene Editing Targeting for Improved T Cell Adoptive Immunotherapy. Mol Ther Nucleic Acids, 2017. 9 : p. 312-321.

20. Kormann, M.S., et al., Expression of therapeutic proteins after delivery of chemically modified mRNA in mice. Nat Biotechnol, 2011. 29 (2): p. 154-7.

21. Dreyer, A.K., et al., TALEN-mediated functional correction of X-linked chronic granulomatous disease in patient-derived induced pluripotent stem cells. Biomaterials, 2015. 69 : p. 191-200.

22. Cornu, T.I. and T. Cathomen, Targeted genome modifications using integrase-deficient lentiviral vectors. Mol Ther, 2007.15 (12): p. 2107-13.

23. Lusso, P., et al., Growth of macrophage-tropic and primary human immunodeficiency virus type 1 (HIV-1) isolates in a unique CD4+ T-cell clone (PM1): failure to downregulate CD4 and to interfere with cell-line-tropic HIV-1. J Virol, 1995. 69 (6): p. 3712-20.

24. Koyanagi, Y., et al., Dual infection of the central nervous system by AIDS viruses with distinct cellular tropisms. Science, 1987.236 (4803): p. 819-22.

25. Adachi, A., et al., Production of acquired immunodeficiency syndrome-associated retrovirus in human and nonhuman cells transfected with an infectious molecular clone. J Virol, 1986. 59 (2): p. 284-91.

26. Kok, Y.L., et al., Monocyte-derived macrophages exhibit distinct and more restricted HIV-1 integration site repertoire than CD4(+) T cells. Sci Rep, 2016. 6 : p. 24157.

27. Moore, J.P., et al., Dissociation of gp120 from HIV-1 virions induced by soluble CD4. Science, 1990. 250 (4984): p. 1139-42.

28. Fine, E.J., et al., An online bioinformatics tool predicts zinc finger and TALE nuclease off-target cleavage. Nucleic Acids Res, 2014. 42 (6): p. e42.

29. Clement, K., et al., CRISPResso2 provides accurate and rapid genome editing sequence analysis. Nat Biotechnol, 2019. 37 (3): p. 224-226. 
30. Benjamini, Y. and Y. Hochberg, Controlling the False Discovery Rate: A Practical and Powerful Approach to Multiple Testing. Journal of the Royal Statistical Society: Series B (Methodological), 1995.57 (1): p. 289-300.

31. Mar, J.C. and J. Quackenbush, Decomposition of gene expression state space trajectories. PLoS Comput Biol, 2009. 5 (12): p. e1000626.

32. Cormier, E.G., et al., Mapping the determinants of the CCR5 amino-terminal sulfopeptide interaction with soluble human immunodeficiency virus type 1 gp120-CD4 complexes. J Virol, 2001.75 (12): p. 5541-9.

33. Dragic, T., et al., Amino-terminal substitutions in the CCR5 coreceptor impair gp120 binding and human immunodeficiency virus type 1 entry. J Virol, 1998. 72 (1): p. 279-85.

34. Meijerink, H., et al., The number of CCR5 expressing CD4+T lymphocytes is lower in HIV-infected long-term non-progressors with viral control compared to normal progressors: a cross-sectional study.BMC Infect Dis, 2014. 14 : p. 683.

35. Bae, S., et al., Microhomology-based choice of Cas9 nuclease target sites. Nat Methods, 2014. 11 (7): p. $705-6$.

36. Tsai, S.Q., et al., GUIDE-seq enables genome-wide profiling of off-target cleavage by CRISPR-Cas nucleases. Nat Biotechnol, 2015.33 (2): p. 187-197.

37. Mock, U., et al., mRNA transfection of a novel TAL effector nuclease (TALEN) facilitates efficient knockout of HIV co-receptor CCR5. Nucleic Acids Res, 2015. 43 (11): p. 5560-71.

38. Qi, C., et al., Inducing CCR5Delta32/Delta32 Homozygotes in the Human Jurkat CD4+ Cell Line and Primary CD4+Cells by CRISPR-Cas9 Genome-Editing Technology. Mol Ther Nucleic Acids, 2018. 12 : p. 267-274.

39. Xiao, Q., et al., CCR5 editing by Staphylococcus aureus Cas9 in human primary CD4(+) T cells and hematopoietic stem/progenitor cells promotes HIV-1 resistance and CD4 ++ ) T cell enrichment in humanized mice. Retrovirology, 2019. 16 (1): p. 15.

40. Shi, B., et al., TALEN-Mediated Knockout of CCR5 Confers Protection Against Infection of Human Immunodeficiency Virus. J Acquir Immune Defic Syndr, 2017. 74 (2): p. 229-241.

41. Xu, L., et al., CRISPR-Edited Stem Cells in a Patient with HIV and Acute Lymphocytic Leukemia. N Engl J Med, 2019. 381 (13): p. 1240-1247.

42. Kordelas, L., et al., Shift of HIV tropism in stem-cell transplantation with CCR5 Delta32 mutation. N Engl J Med, 2014.371 (9): p. 880-2.

43. Didigu, C.A., et al., Simultaneous zinc-finger nuclease editing of the HIV coreceptors ccr5 and cxcr4 protects CD4 + T cells from HIV-1 infection. Blood, 2014. 123 (1): p. 61-9.

44. Liu, Z., et al., Genome editing of the HIV co-receptors CCR5 and CXCR4 by CRISPR-Cas9 protects CD4(+) T cells from HIV-1 infection.Cell Biosci, 2017. 7 : p. 47.

45. Yu, S., et al., Simultaneous Knockout of CXCR4 and CCR5 Genes in CD4+ T Cells via CRISPR/Cas9 Confers Resistance to Both X4- and R5-Tropic Human Immunodeficiency Virus Type 1 Infection. Hum Gene Ther, 2018. 29 (1): p. 51-67.

46. Lim, J.K., et al., CCR5 deficiency is a risk factor for early clinical manifestations of West Nile virus infection but not for viral transmission. J Infect Dis, 2010. 201 (2): p. 178-85.

Figures 
A

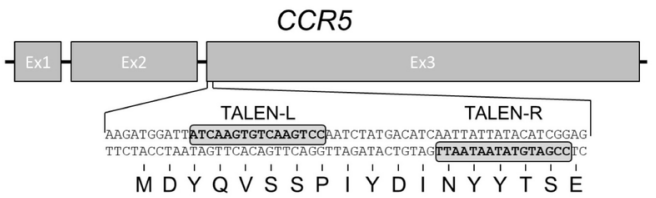

B

D
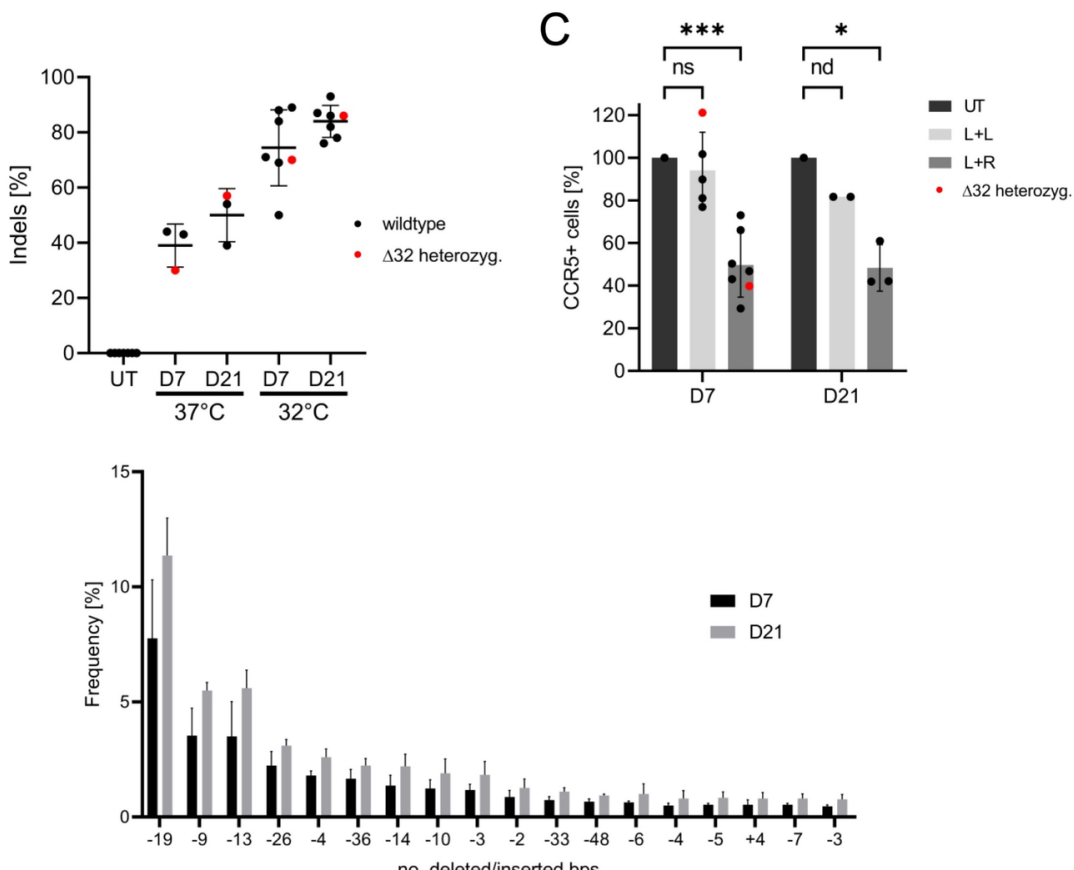

$\mathrm{E}$

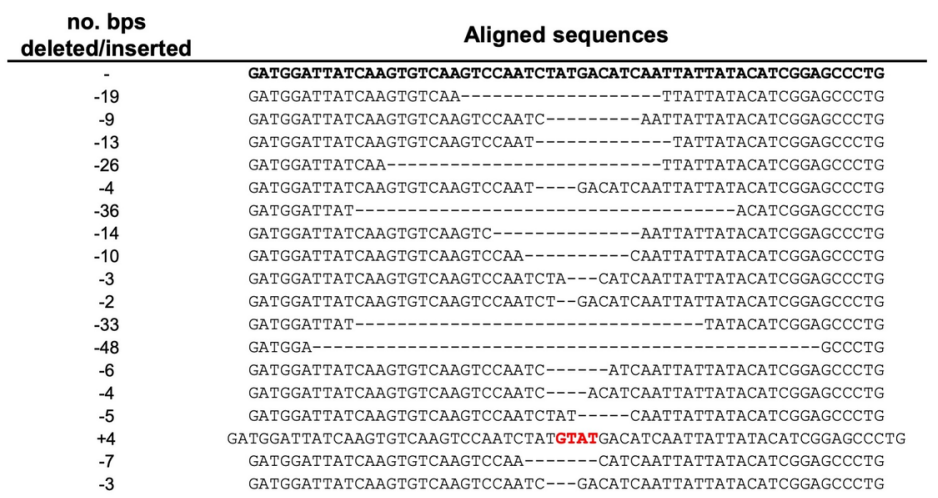

Figure 1. Stable knockout of $C C R 5$ in CD4+ T cells mediated by TALEN. A) Schematic of the $C C R 5$ locus with TALEN binding sites in exon 3 (boxes). B) Quantification of TALEN activity. Multiple T7E1 assays were performed in CD4+ T cells of 7 or 3 different donors at day $7 / 21$ post-electroporation. Where indicated, cells were transiently incubation at $32^{\circ} \mathrm{C}$ for $24 \mathrm{~h}$, or kept at $37^{\circ} \mathrm{C}$, respectively. Samples derived from a $\Delta 32$ heterozygous donor are highlighted in red $(\mathrm{n}=3-7 ; \pm \mathrm{SD})$. C) Expression of endogenous CCR5 in CD4+ T cells. Cell were treated with only left TALEN arm $(\mathrm{L}+\mathrm{L})$ or both TALEN arms $(\mathrm{L}+\mathrm{R})$. Data is shown relative to untreated (UT) cells. Each dot represents a different donor (n=2-7; \pm SD; ns=not significant, 
nd $=$ not determined, ${ }^{*} p[?] 0,05 ; * * * p[?] 0,001$, paired $t$-test). D) Frequency of various mutations. Targeted amplicon sequencing of the target site was performed after 7 and 21 days of culture in 3 different donors $(\mathrm{n}=3$; +-SD). Displayed are the frequencies of all indels with a frequency $>1$ in at least one of the three experiments. E) Sequence of induced mutations. Displayed are all sequences quantified in (D).

A

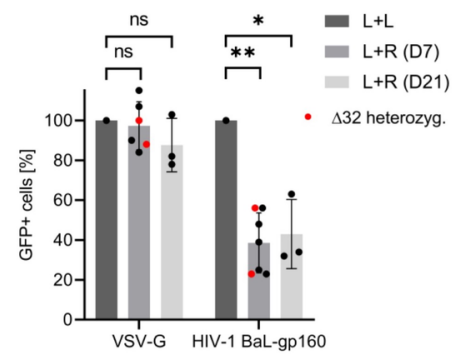

C

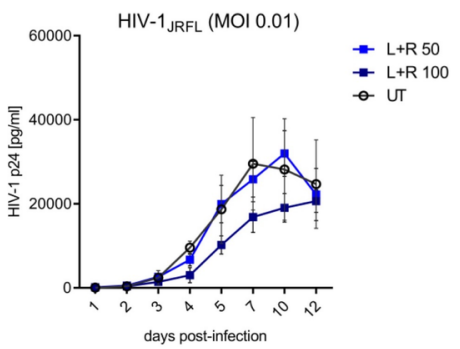

B

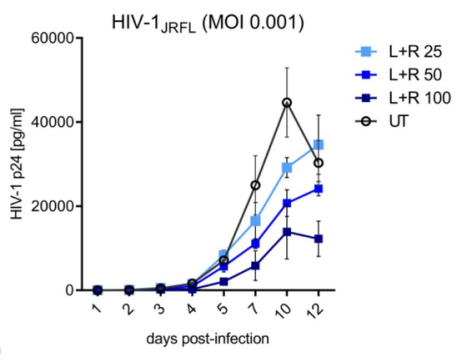

D

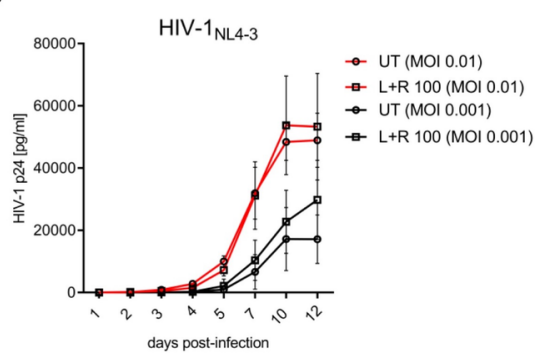

Figure 2. CCR5 disruption confers HIV-1 resistance. A) Lentiviral challenge. Untreated (UT) cells as well as TALEN edited cells $(\mathrm{L}+\mathrm{L} ; \mathrm{L}+\mathrm{R})$ were transduced with GFP-expressing lentiviral particles that were either pseudotyped with VSV-G or HIV-1BaL-gp160. Challenge was performed at day 7 and day 21 of culture. Indicated is the percentage of GFP-positive cells relative to the corresponding untreated cells. Samples derived from two $\Delta 32$ heterozygous donor are marked in red. Each dot represents a different donor ( $\mathrm{n}=3-7$; $\pm \mathrm{SD}$; ns $=$ not significant, ${ }^{*} p[?] 0,05 ;{ }^{* *} p[?] 0,01$, paired $t$-test). B-D) Challenge with HIV-1. Shown is the p24 kinetics over the course of 12 days of infection in untreated (UT), TALEN edited cells (L+R 100), and edited cells spiked with UT at ratios of 1:1 (L+R 50) or 1:3 (L+R 25). Cells were challenged with HIV-1 JRFL at an MOI of 0.001 (B), an MOI of 0.01 (C) or with HIV-1 $1_{\mathrm{NL} 3-4}$ at an MOI of 0.01 and 0.001 in triplicates $+-\mathrm{SD}(\mathrm{D})$. 
A

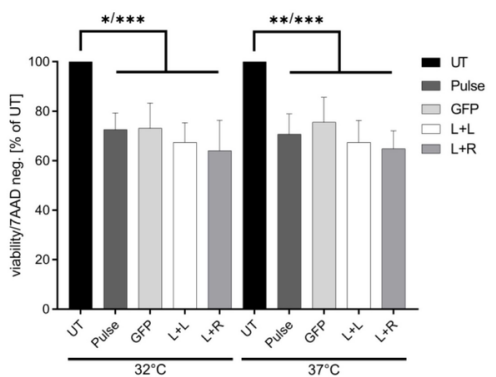

C

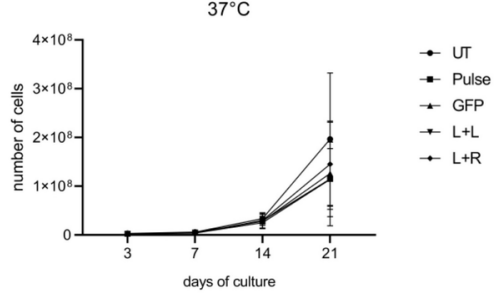

$\mathrm{E}$

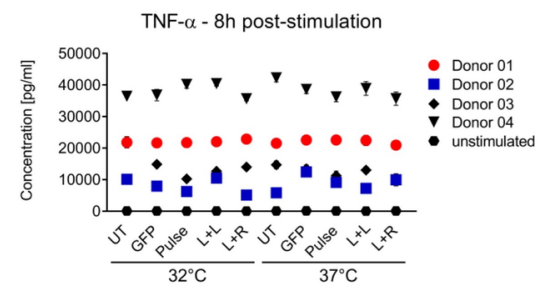

B

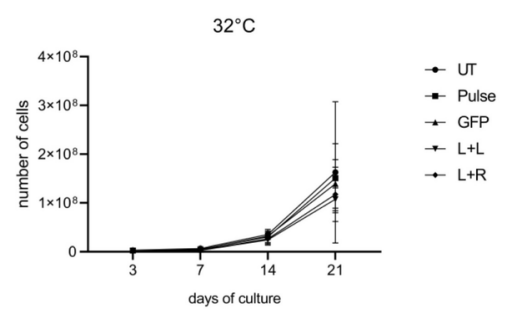

D

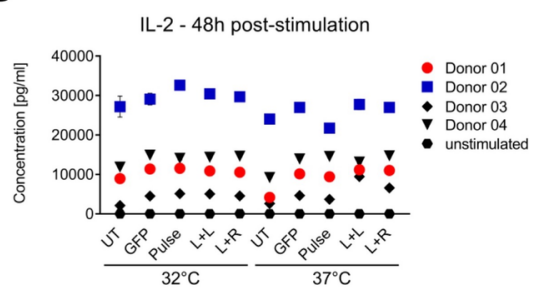

F

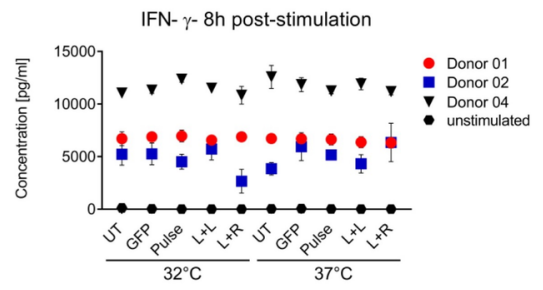

Figure 3. CCR5 knockout does not impair CD4+ T cell functions. A) Viability. Shown is the viability of CD4+ T cells of several donors $24 \mathrm{~h}$ after they were either mock-electroporated (Pulse), or electroporated with mRNA (GFP/TALEN L+L/TALEN L+R) relative to untreated (UT) cells $\left(\mathrm{n}=4-12 ;{ }^{*} p[?] 0,05 ;{ }^{* *} p\right.$ $\left.[?] 0,01 ;{ }^{* * *} p[?] 0,001 ; \pm \mathrm{SD}\right)$. B-C) Proliferation. Shown are the growth curves of CD4+ T cells subjected to transient cold-shock at $32^{\circ} \mathrm{C}(\mathrm{B})$ or kept at $37^{\circ} \mathrm{C}(\mathrm{C})$ after being mock-electroporated (Pulse) or electroporated with mRNA (GFP, TALEN arms $\mathrm{L}+\mathrm{L} / \mathrm{L}+\mathrm{R}$ ), or left untreated (UT). Cells were counted at the indicated time points. Error bars represent \pm SD. D-F) Cytokine secretion. Cytometric bead array was used to assess the release of TNF- $\alpha$ (D), IFN- $\gamma(\mathrm{E})$ or IL-2 (F) at indicated timepoints after stimulation. Samples were measured in triplicate, error bars represent $\pm \mathrm{SD}$. 
A
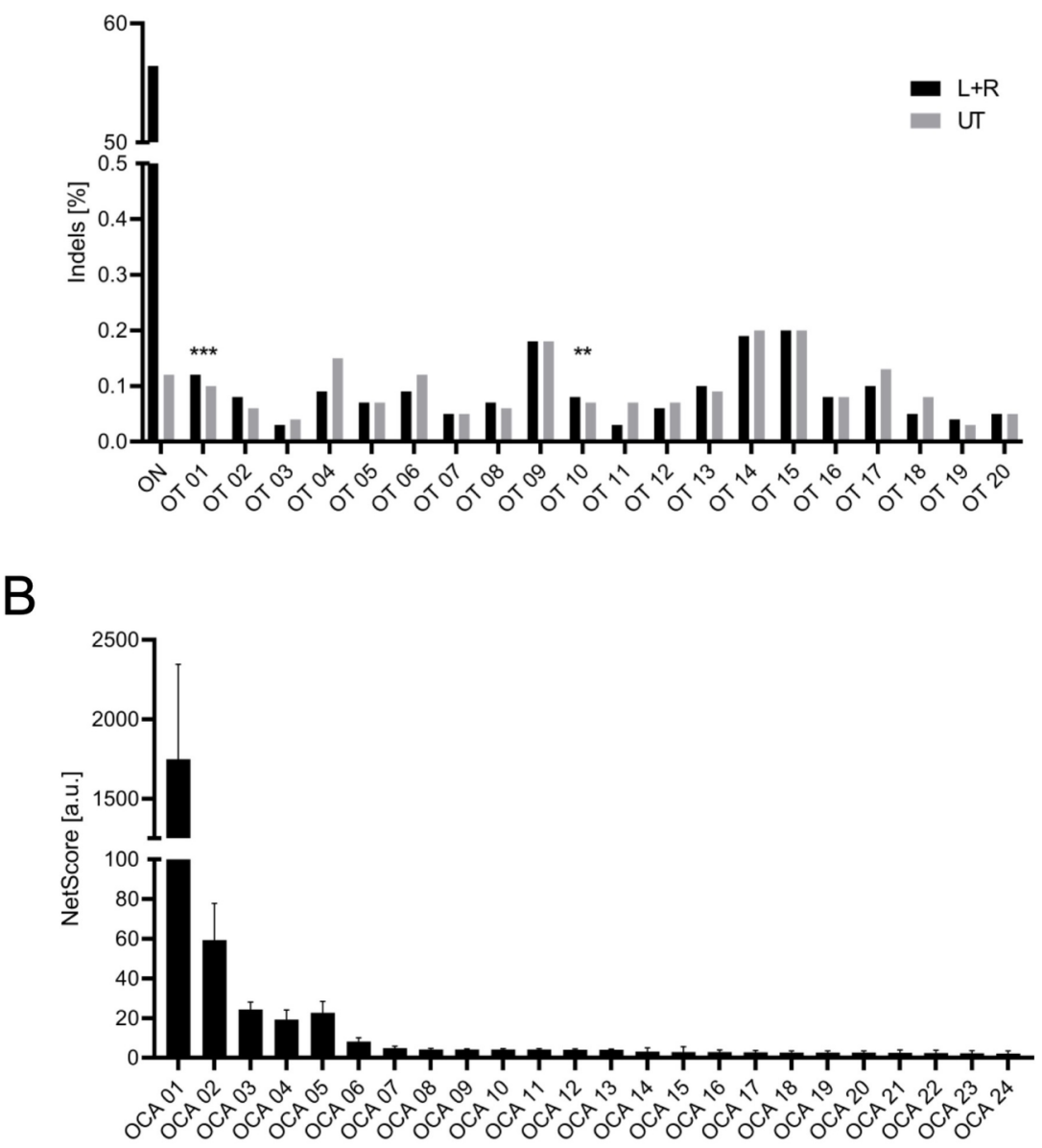

Figure 4. Off-target analysis in TALEN edited CD4+ T cells. A) Targeted amplicon sequencing of the top 20 off-targets predicted by PROGNOS and analyzed by Cripresso2 (** $\left.p[?] 0,01,{ }^{* * *} p[?] 0,001\right)$. B) Oligonucleotide-capture assay (OCA) performed in $\mathrm{CD} 4+\mathrm{T}$ of three different donors and measured in triplicate. Bars represent the mean \pm SD of all nine measurements after subtraction of the scores obtained for mock-treated cells. 